\title{
La Investigación en la Enseñanza Universitaria de Química: un Caso en la Formación Inicial Docente
}

\author{
Research in University Teaching of Chemistry: A Case in the Faculty's Initial Teaching \\ A pesquisa no ensino universitário de Química: um caso de Formação Inicial Docente
}

Zenahir Siso-Pavón* https://orcid.org/0000-0002-0523-6392

Departamento de Didáctica. Facultad de Educación. Universidad Católica de la Santísima Concepción, Chile

$\nabla$

Recibido: 05-12-17 Revisado: 07-06-18 Aceptado: 05-08-18 Publicado: 20-12-18

- Resumen. Con el propósito de caracterizar los procesos de investigación promovidos en la enseñanza universitaria de la Química en la formación inicial del profesorado de esta disciplina, se realizó la observación participante de las clases de cursos dictados por cuatro docentes del área de Química Orgánica de una universidad venezolana. Emergieron categorías de análisis e interpretación sobre las que se indagó a través de una entrevista retrospectiva y se aplicó una entrevista semiestructurada a doce estudiantes de estos cursos. Las categorías resultantes fueron (a) La investigación como proceso, (b) Planteamiento de situaciones problemáticas y (c) Estudio cualitativo de las situaciones problemáticas, estudiándose el concepto de investigación de los docentes y su aplicación en la praxis docente, consideración de ideas, destrezas y actitudes, el uso de la bibliografía, la emisión de hipótesis, la elaboración de estrategias de solución y el manejo de los nuevos conocimientos. Se encontró que la investigación se entiende principalmente como la indagación bibliográfica con relación a los temas, que permite los abordajes teóricos y de trabajos prácticos sin una mayor elaboración de hipótesis y de estrategias de resolución, por cuanto se tratan de trabajos altamente dirigidos con débil contribución a la promoción de habilidades científicas.
Palabras clave:

Investigación,

Habilidad,

Docencia

Universitaria,

Formación Inicial

Docente, Química 
Abstract. With the purpose of characterizing the research processes promoted in the university teaching of Chemistry in the initial training of the teaching staff of this discipline, the participant observation was made of the classes of courses taught by four teachers of the area of Organic Chemistry of a Venezuelan university. Categories of analysis and interpretation emerged, which were researched through a retrospective interview; also, a semi-structured interview was applied to twelve students of these courses. The resulting categories were (a) Research as a process, (b) Proposal of problematic situations, and (c) Qualitative study of problem situations, studying the concept of teacher research and its application in teaching practice, consideration of ideas, skills, and attitudes; the use of bibliography, the issuance of hypotheses; the elaboration of solution strategies, and management of new knowledge. It was found that research is mainly understood as the bibliographic inquiry in relation to topics, which allows theoretical and practical approaches without further elaboration of hypotheses and resolution strategies because they are highly directed work with little contribution to the promotion of scientific skills.

- Resumo. Com o intuito de caracterizar os processos de pesquisa promovidos no ensino universitário de Química na formação inicial dos professores desta disciplina, realizou-se a observação participante de aulas de cursos ministrados por quatro professores da área de Química Orgânica de uma universidade venezuelana. Surgiram categorias de análise e interpretação sobre as que se indagou por meio de uma entrevista retrospectiva e aplicou-se uma entrevista semiestruturada a doze estudantes de tais cursos. As categorias resultantes foram (a) a pesquisa como processo, (b) apresentação de situações problemáticas, e (c) o estudo qualitativo das situações problemáticas com foco no estudo do conceito de pesquisa por parte dos professores e a sua aplicação na prática docente, consideração de ideias, destrezas e atitudes, o uso da bibliografia, a formulação de hipótese, a elaboração de estratégias de solução e a gestão de novos conhecimentos. O estudo revelou que a pesquisa é entendida, principalmente, como uma indagação bibliográfica sobre os temas, que permite abordagens teóricas e de trabalhos práticos sem formulação de hipótese e de estratégias de resolução por se tratarem de trabalhos altamente dirigidos e com pouca contribuição na promoção de habilidades científicas.
Keywords:

research, skill, university teaching, teacher's initial training, chemistry

Palavras-chave:

Pesquisa, Habilidade, Docência Universitária, Formação Inicial Docente, Química 
a formación del profesorado de ciencias que se desempeña en los niveles de educación básica y media ha constituido un punto focal de interés durante las últimas tres décadas, en las que se ha reconocido como profesional reflexivo, alejándose de la perspectiva de racionalidad técnica (Mellado \& González, 2000). Este énfasis en el pensamiento del profesor, quien transforma el conocimiento de la asignatura en representaciones comprensibles, lo hace constructor de un cuerpo de conocimiento sobre la base de sus concepciones, roles, conocimientos, actitudes y experiencias vivenciadas durante la formación inicial, determinado por los conocimientos profesionales para aprender a enseñar Ciencias (Couso, 2002; Cuéllar, 2010; García, 2009; Vázquez, Jiménez \& Mellado, 2007)

Estos conocimientos son saberes epistemológicamente diferentes que evolucionan y se constituyen como un gran cuerpo de conocimientos, entre el que se encuentra el contenido de ciencias, que se refiere tanto a lo sustantivo y lo sintáctico de ella; es decir, el conocimiento teórico, los marcos teóricos y la estructura interna en una parte; y en la otra, la fundamentación y evolución de los paradigmas, la historia-filosofía y naturaleza de las ciencias (Adúriz-Bravo, 2011; Garritz \& Velasco, 2004; Pozo \& Gómez, 2013; Vázquez \& Manassero, 2012). Este conocimiento científico del profesor de ciencias se organiza a través de sus experiencias de aprendizaje, las que en Química como ciencia experimental involucran al laboratorio como un espacio donde el docente propicia el desarrollo de habilidades de investigación, a la vez que las interacciones entre la teoría y la práctica.

Ante el progresivo cuestionamiento de la enseñanza y de sus métodos que no corresponden a las necesidades personales ni al progreso de las ciencias y el desarrollo social, se mueven algunas fuerzas que apuntan a la renovación de las estructuras y contenidos de la educación. Desde esta perspectiva, se considera relevante la práctica e investigación por el cambio de posición de un enfoque centrado en la enseñanza, a otro que considere significativamente al aprendizaje y que introduzca modificaciones en cuanto a su sentido y propósito, ya que más que acumular conocimientos que se superen rápidamente ha de dirigirse al desarrollo de las habilidades, que coloque al individuo en condiciones de ser capaz de autodirigirlo y autogestionarlo conforme a sus propios intereses necesidades individuales y colectivas.

Además, son los docentes de una institución los que deben admitir que la investigación científica debe ser transversal a los procesos de Enseñanza y Aprendizaje, alimentando el progreso de la educación, ya que si se entiende como elemento vinculador de la práctica y la reflexión docente, por medio de ella se ejercitará en cada unidad curricular integrante del plan de estudios la capacidad para analizar información, confrontar resultados con otras investigaciones y conceptos consolidados e introducir innovaciones en las prácticas educativas. Así, el fin de la investigación no sería predecir y controlar, sino interpretar y comprender fenómenos. 


\section{La investigación como estrategia}

Desde una postura reduccionista, la experimentación que caracteriza a la investigación científica disciplinar y a las aproximaciones investigativas desarrolladas en el aprendizaje científico, se ajustan al mero trabajo de laboratorio y descuidan aspectos fundamentales del desarrollo del conocimiento. En su trabajo clásico, ya Gil (1993) señalaba que este último debe constituir el eje de la investigación, por cuanto la enseñanza y el aprendizaje de las ciencias como investigación se refiere al abordaje de problemas de interés que coadyuven al estudiante a construir su conocimiento desde la revisión bibliográfica, pasando por experimentación en laboratorios hasta lograr interpretar modelos conceptuales que permitan explicar los fenómenos observados, de forma que el estudiante asuma la posición de un investigador (Gil, 1993; Pozo \& Gómez, 2013), por lo que resulta favorable orientar el aprendizaje de las Ciencias a través de la investigación y de situaciones problemáticas (Belmonte, 2011; Burbules \& Linn, 1991; Caamaño, 2012)

La enseñanza por investigación en torno a problemas ha sido caracterizada por JiménezTenorio y Oliva (2016) como una estrategia que invita a superar el cambio conceptual, en la que confluyen variedad de propuestas, desde el llamado enfoque por indagación a la investigación dirigida y que asume la existencia de distintas fases del trabajo científico, "pero entendido ahora desde perspectivas más acordes con las ideas actuales sobre la naturaleza de la ciencia: necesidad de un problema, existencia de un conocimiento teórico previo, el papel de las hipótesis y los procesos de diseño de investigación", entre otras (p. 124)

La posición de "investigador novel" sería asumida por los estudiantes con el acompañamiento del docente -quien actúa a modo de guía en la resolución del problema planteado-, lo que supone una serie de ventajas para el proceso de aprendizaje de los estudiantes a través de la investigación, cuestión relevante en la formación inicial del profesorado. Al respecto, Ungerfeld (2004) plantea las siguientes:

1. Utilización del pensamiento lógico y desarrollo de la capacidad para interpretar situaciones, ya que es necesario ser conscientes de la raíz o génesis de los problemas para trabajarlos reiteradamente, elaborando alternativas posibles.

2. Desarrollo de diseños que permitan solucionar problemas, pues el participar en actividades investigativas, permite aprender sobre la viabilidad de discurrir en una u otra vía para llegar a la solución.

3. Aprender a recombinar conocimientos, para explicar una realidad que ha sido previamente segmentada en aras de su estudio, llevando a la incomprensión de fenómenos complejos.

4. Desarrollo de aptitudes intuitivas, que lolleven a intuir respuestas generando hipótesis.

5. Desarrollo de capacidades propias, de auténticas formas de aprender.

6. Desarrollo del pensamiento crítico donde el estudiante construya sus argumentos científicos, trascendiendo el positivismo reflejado en las conclusiones directas y las 
respuestas asumidas como verdades.

7. Utilización de un lenguaje adecuado, que se logra a través de la lectura e indagación y que permite la producción de textos y su comunicación con la comunidad científica.

8. Desarrollo de la curiosidad por cuanto la investigación genera el deseo de "saber más".

Puede entenderse entonces que la investigación potencia en los estudiantes procesos de pensamiento de orden superior, además de la oportunidad de transferir conocimientos a situaciones diferentes a las que originaron inicialmente el aprendizaje. Atendiendo a lo anterior, se contemplan cuatro fases de la investigación como estrategia para el logro del aprendizaje (ver Figura 1).

Esta noción de la investigación como estrategia sirve de base orientadora al presente estudio, ya que sustenta lo abordado en relación con la construcción de conocimientos en los cursos disciplinares de la formación del profesorado de ciencias, en particular Química, donde es tan importante comprender los fenómenos y reelaborarlos para poder generalizar y transferir a otras situaciones.

Plantear el manejo reiterado de los nuevos conocimientos en variedad de situaciones

Concepción de nuevos problemas

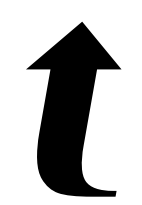

Orientar el tratamiento científico de los problemas planteados: Resolución y Análisis

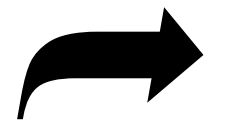

Plantear situaciones problemáticas

\section{La investigación} como Estrategia

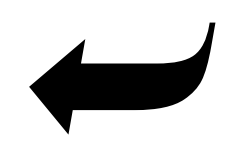

Proponer el estudio cualitativo de las situaciones problemáticas planteadas

Figura 1. La Investigación como Estrategia. Elaborada con datos tomados de Gil (1993)

\section{El Laboratorio de Ciencias y la Investigación}

Con las reformas de la enseñanza de las ciencias en los años sesenta, la idea predominante era que los laboratorios no debían basarse en simples demostraciones o verificaciones de información conocida, sino que debe centrarse en la resolución de problemas, en el desarrollo de destrezas necesarias para investigar y para brindar a los estudiantes la oportunidad de descubrir.

- Partiendo del carácter experimental de la química, el espacio de laboratorio es un elemento esencial ya que proporciona al educando la posibilidad de imbuirse en los 
procesos de la ciencia, utilizando la experimentación como un conjunto de actividades destinadas a conceptualizar los datos empíricos, situándose en las perspectivas actuales de la enseñanza de las ciencias. Sin embargo, muchos autores han criticado el rol del laboratorio en la enseñanza de la Química, y al respecto, García (2000) presenta una síntesis de tales posturas Las clases experimentales en líneas generales están excesivamente cargadas, lo que implica que los estudiantes tengan muy poca responsabilidad de sus aprendizajes y piensen muy poco por sí mismos

- Los docentes enfatizan en las respuestas correctas, por lo que el laboratorio tiende a la verificación de cosas conocidas

- El trabajo de laboratorio es un trámite puramente académico, en donde se realizan distintas experiencias aparentemente inconexas y sin aplicación alguna

- La cantidad de instrucciones que el estudianterecibees uno delos elementos que impiden el logro del verdadero aprendizaje, ya que al realizar las actividades experimentales ejecutan tales instrucciones sin comprender lo que han hecho

- La forma confusa y nada productiva de los trabajos prácticos les confiere poco valor educativo

- En los laboratorios se hacen verificaciones, las cuales son hechas por los estudiantes guiándose como si fuera una receta de cocina

Estas críticas evidencian entonces una necesidad de cambiar el cuadro, de redimensionar lo que en el laboratorio se hace. Muchos son los acuerdos a nivel global de que el objetivo de las clases experimentales es el desarrollo en los estudiantes de habilidades, destrezas y hábitos, por cuanto se deben enseñar destrezas para la adquisición (estrategias de aprendizaje) y aplicación de los conocimientos impartidos (solución de problemas) desde una perspectiva investigativa. Por ello, como objetivo de este estudio, se propuso caracterizar los procesos de investigación promovidos en la enseñanza universitaria de la Química en la formación inicial del profesorado de esta disciplina.

\section{MÉTODO}

\section{Diseño}

El estudio, de tipo cualitativo, se desarrolló bajo el paradigma interpretativo-constructivo (Álvarez-Gayou, 2009; Dezin \& Lincoln, 2012; Flick, 2004), considerándose para su desarrollo, un estudio de caso múltiple (Neiman \& Quaranta, 2006).

\section{Participantes}

El campo de estudio estuvo constituido por universidades formadoras de profesores de Química de la zona central de Venezuela, y los participantes clave fueron seleccionados por muestreo completo, el que permite "definiciones a priori de la estructura de la muestra" y "delimitan la totalidad de casos posibles de manera que todos se pueden integrar en el estudio" (Flick, 2004, p. 
77), atendiendo a razones teóricas y buscando un conjunto de casos que permitieran dar cuenta del fenómeno por analizar. Por ello, los siguientes son los criterios inicialmente considerados:

- Homogeneidad: todos los docentes deben ejercer en el nivel universitario, cuyo lugar de trabajo sea el aula y laboratorio de Química, del Departamento de Química. Género hombre/mujer

- Heterogeneidad: los docentes pueden tener formación inicial de Licenciado o Profesor, así como estar en prediplomado o especialización, y/o grado de cuarto nivel (Maestría y/o Doctorado). También pueden impartir cualquier asignatura vinculada con el área de conocimiento de química orgánica

- Inclusión: se incluyen en el estudio docentes con tres o más años de experiencia en docencia universitaria.

Luego, se seleccionaron cuatro participantes por razones instrumentales (Simons, 2011) sobre la base de la voluntariedad manifiesta y aceptación de participación. Estos docentes fueron todos del área de conocimiento Química Orgánica, identificados con los nomencladores Docente informante A (DI-A), Docente informante B (DI-B), Docente informante C (DI-C), Docente informante $D(D I-D)$ con las siguientes características:

- DI-A; DI-B y DI-D: Profesores en la Especialidad de Química. Magíster en Educación mención Enseñanza

- DI-C: Licenciado en Química. Candidato a Magíster en Educación.

\section{Instrumentos}

Respecto de las técnicas e instrumentos de recolección de información, se puntualiza lo siguiente: Observación participante

De acuerdo con lo observado en las clases de los docentes participantes, emergieron las categorías que orientaron el trabajo investigativo empleando una bitácora-cuaderno de notas. Esta técnica, según Couso (2002) permite alternar el grado de observación y participación según el momento en el que sea requerido. El investigador entra al campo a producir los datos y reflexiona sobre lo que ocurre, en función de lo que se quiere observar.

Entrevista retrospectiva a los docentes informantes clave

Se diseñó un guion de preguntas basándose en las categorías y subcategorías emergidas de las observaciones realizadas a las clases teóricas y prácticas, como se mencionó anteriormente. La información fue transcrita a textos, constituyendo así unidades de análisis, que posteriormente fueron sintetizadas para su estudio.

Entrevista no estructurada a los estudiantes informantes clave

Con el fin de indagar sobre la investigación como estrategia de enseñanza de los docentes informantes clave, hubo la necesidad de conocer una tercera perspectiva: la del estudiante. Para 
ello, durante el periodo de la observación participante, se seleccionaron tres (3) estudiantes por cada docente, a quienes se les solicitó la colaboración como estudiantes informantes clave para la presente investigación. Así, se aplicó una entrevista semiestructurada (Canales, 2006; Simons, 2011) en relación con una lista de cuestiones relativas sustentada en lo observado previamente en las clases.

\section{Procedimiento}

Para el logro del objetivo planteado en este estudio, y tras la observación participante, emergió el sistema de categorías y subcategorías (ver Tabla 1) sobre las que se generaron las preguntas de las entrevistas retrospectivas y semiestructuradas.

El análisis de la información se desarrolló a través del análisis de contenidos, como un conjunto de procedimientos interpretativos de productos comunicativos (mensajes, textos o discursos) que proceden de procesos singulares de comunicación previamente registrados, y que, basados en técnicas de medida, tienen por objeto elaborar y procesar datos relevantes sobre las condiciones mismas en que se han producido los textos y que funciona por procedimientos sistemáticos y objetivos de descripción del contenido de los mensajes.

Tabla 1.

Categorías y Subcategorías del estudio

\section{La investigación como proceso}

Marco de acción que involucra al docente y al estudiante mientras se desarrollan los contenidos curriculares correspondientes a la asignatura.

\section{Planteamiento de situaciones problemáticas}

Acciones docentes estructuradas y planificadas en forma secuencial y orientadas a fines específicos

\section{Estudio cualitativo de las situaciones problemáticas}

Evaluación y planteamiento de vías de resolución

\section{Concepto de investigación y su aplicación en la praxis docente}

Marco organizador referencial desde el que se entiende a la investigación y se actúa en consecuencia desde el rol docente

\section{Consideración de ideas, destrezas y actitudes}

Involucramiento que hace el docente de los aportes y habilidades de los estudiantes a la acción investigativa

- Uso de la bibliografía

- Emisión de hipótesis

- Elaboración de estrategias de solución

- Manejo de los nuevos conocimientos

Habilidades de pensamiento científico promovidas a través de la evaluación y planteamiento de vías de resolución 
La información provista en los cuestionarios y en las entrevistas semiestructuradas adoptó el modelo propuesto por Canales (2006) donde se hace alusión al proceso de análisis estructural del discurso, el cual incluye un primer momento de recomposición de las estructuras, donde se identifican las unidades básicas de sentido y las relaciones entre ellas; un segundo momento de dinamización de las estructuras donde se organizan las relaciones encontradas en un modelo de acción que permite orientar la práctica de los sujetos; y por último, un momento final donde por triangulación es posible establecer los aspectos emergentes de las reflexiones de acuerdo con la Figura 2.

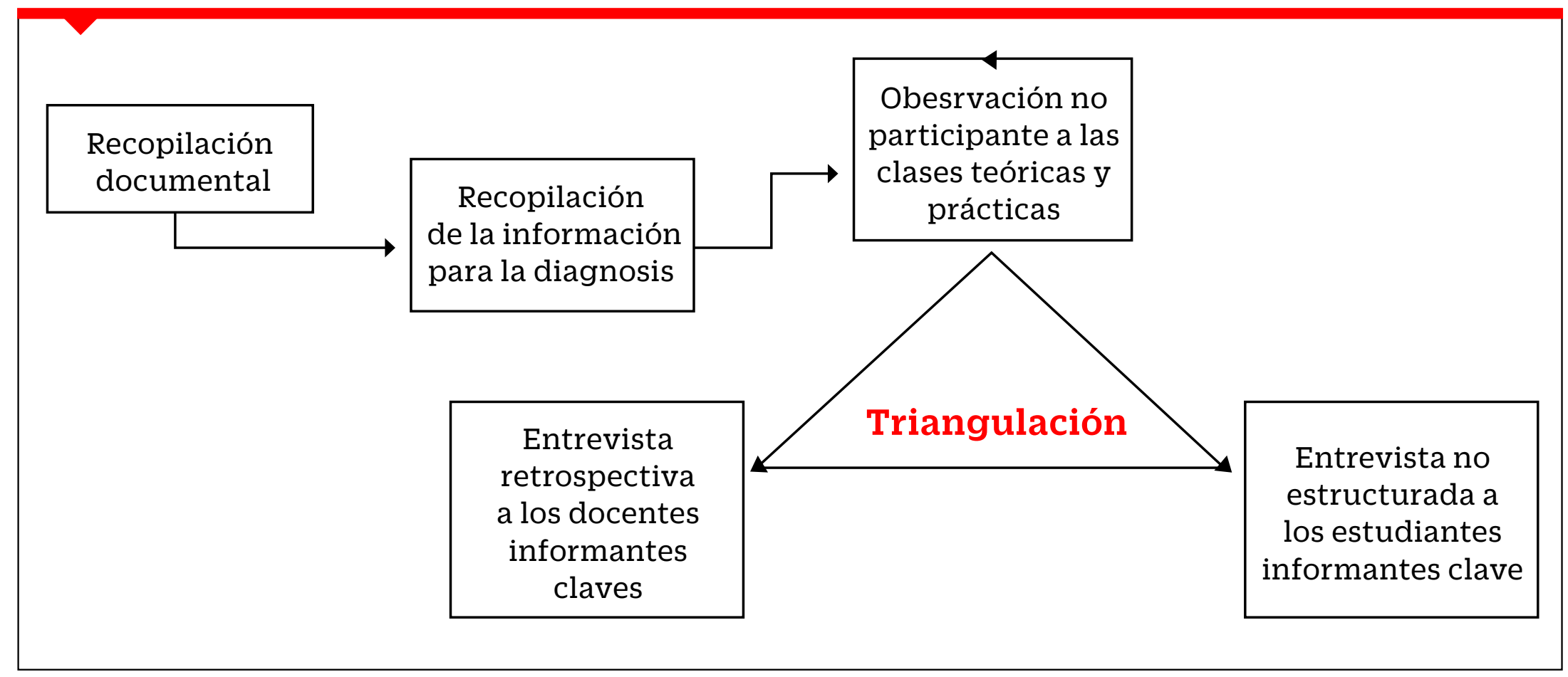

Figura 2. Procedimiento metodológico

\section{RESULTADOS}

Luego de la reducción de los datos, correspondiente codificación y categorización de la información producida a través de la observación y las entrevistas realizadas a los docentes y estudiantes informantes, se procedió a la triangulación de la información por cada categoría y subcategoría en estudio, lo que se muestra tras una previa discusión, a continuación.

\section{Concepto de investigación y su aplicación en la praxis docente}

Para los docentes informantes clave, la investigación está conceptualizada como un proceso de búsqueda e indagación de información, que además se considera fundamental para el aprendizaje y la consolidación del conocimiento. Sin embargo, al momento de emplearla en clase, tal proceso se limita a que el estudiante resuelva guías de ejercicio y problemas, lea o revise aspectos teóricos que quedan indicados por el docente en el mejor de los casos, pues 
pueden ocurrir dos cosas: o se puntualiza lo que el estudiante debe leer, o se les permite que lean a rasgos generales sobre el tópico por discutir.

No se apreció a través de la observación en los participantes que los docentes indicaran lectura sobre un problema-contexto. Esto es un aspecto relevante, en tanto los docentes refirieron que la investigación es indispensable en la vida universitaria, cuestión que se condice con los aportes de diversas investigaciones, y relevada por algunos como la forma más perfecta de la enseñanza universitaria (Podestá et al. como se citaron en Ungerfeld, 2004). Este autor refiere también que el fin de la misma debe ser la formación científica y el desarrollo de una mentalidad creadora a nivel universitario, que transite desde el estudiar más, hacia la comprensión orientada desde lo metodológico, el cómo se estudia y el cómo se aprende

Es por esto que surge la pregunta: ¿realmente se está fomentando la investigación en el aula a través de asignación de lecturas y el "mandar a leer" a los estudiantes? La investigación puede incorporarse a la enseñanza a partir deactividades que demanden un mayor compromiso, muy superior al de leer para la próxima clase.

En un orden de ideas similar, esta situación afecta también al espacio de laboratorio, pues los docentes reconocen que en él "no se investiga" y semestralmente se reproducen las prácticas que están contenidas en el manual, información concordante con lo expuesto por los estudiantes y lo observado. Esto permitió conocer que las prácticas que se realizan son de comprobación de la teoría. Sin embargo, muchas veces los contenidos tratados en laboratorio son antiparalelos a los tratados en la clase teórica.

En la Figura 3 se evidencian los elementos triangulados a partir de las tres instancias de producción de información.

\section{Consideración de ideas, destrezas y actitudes}

Con relación a este aspecto, cuando el docente plantea situaciones problemáticas tomando en cuenta la visión de mundo, destrezas y actitudes de sus estudiantes, las mismas pueden generar un interés y una concepción preliminar de la tarea por desarrollar (Gil, 1993). Sin embargo, la forma en la que los docentes informantes clave consideran estas ideas, destrezas y actitudes es escuchando la vía de resolución que los estudiantes alcanzan a enunciar una vez que ya se ha planteado el problema en la clase teórica -que muchas veces se trata de la resolución de ejercicios numéricos y algorítmicos, repetitivos y cerrados, lo que es una tradicional visión de la enseñanza de la química que impide la adecuada interpretación de la resolución de problemas como estrategia - y, a nivel experimental reconocen el temor que sienten cuando un estudiante plantea una idea para llevarla a cabo en el laboratorio, puesto que generalmente lo hace por curiosidad y sin sustento o aval teórico. 


\section{Observación no participante}

- Los estudiantes investigan cuando leen para la próxima clase lo que se va a discutir en ella, pues se tiende a asignar la revisión sobre diversos aspectos teóricos para discutir la clase siguiente. Esto se hace de manera puntual sobre el contenido, y no sobre un contexto-problema específico.

- Las prácticas de laboratorio son de verificación de la teoría. La investigación se caracteriza por comprender el por qué de los hechos experimentales ya preestablecidos.

\section{Entrevista retrospectiva}

- La investigación es un proceso de indagación y búsqueda de información, el cual es fundamental o indispensable en el aprendizaje, en el desarrollo de la vida universitaria, al momento de consolidar un conocimiento

- La emplean a través de la revisión de los textos antes de la clase o desarrollo de contenido, pues asignan a los estudiantes actividades ponderadas que deben realizar para obtener una calificación, lo que los conduce a investigar, consultar, indagar porque es un contenido que no se ha discutido en el aula.

- En el laboratorio no se no se investiga propiamente hablando, pues solo se ha utilizado el manual de laboratorio que ha existido en las diversas materias.

- La finalidad de hacer una práctica de laboratorio es ampliar lo visto en teoría, comprobar o verificar los hechos teóricos desarrollados en clases y que el estudiante desarrolle habilidades y destrezas dentro del laboratorio a través del abordaje de un contenido

Concepto de

investigación y la praxis docente

\section{Entrevista no estructurada}

- Mandan a investigar, a leer lo que se va a discutir la clase siguiente, a realizar lecturas que trae a la clase y a resolver los problemas de las guías, previa revisión de la teoría

- Las prácticas tienden a demostrar teorías siguiendo los pasos que se encuentran establecidos en las guías de laboratorio y las instrucciones establecidas en ella. No se busca "ir mas allá". Además, las actividades experimentales no se relacionan con lo que se va explicando en las clases teóricas

Figura 3. Categoría: La investigación como proceso. Subcategoría: Concepto de investigación y su aplicación en la praxis docente 
Entonces, he aquí una situación dual: el docente no considera los aportes de los estudiantes en las clases teóricas para proponer problemas y así incrementar el interés y fomentar la participación; sin embargo, cuando estas ideas fluyen en el ámbito experimental, no son consideradas ante la incertidumbre desde las que son planteadas, aun cuando saben que provienen de la curiosidad y por tanto podrían desencadenar una serie de situaciones de aprendizaje realmente auténticas. Lo anterior es advertido por Jiménez-Tenorio y Oliva (2016) cuando señalan que las ideas previas son útiles para dar sentido al problema, así como para la formulación de hipótesis y la orientación de todo el proceso de investigación.

En cuanto al desarrollo de destrezas y actitudes, la información producida revela que no forma parte del discurso de los docentes entrevistados (ver Figura 4), por lo que solo se compendió al respecto que en los laboratorios hay preocupación por indicar a los estudiantes el qué hacer y el cómo proceder. De esta manera se identifica la ausencia del desarrollo de planteamientos de preguntas, de hipótesis de trabajo, de diseños experimentales, de manejo de datos y análisis de los mismos para la interpretación y elaboración de conclusiones, así como la correspondiente revisión metarreflexiva como buena descripción del proceso de investigación en cuanto a lo procedimental y actitudinal en los estudiantes (Ferrés, Marbá \& Sanmartí, 2015).

\section{Observación no participante}

- Casi siempre consideran los planteamientos de los estudiantes en cuanto a la resolución de un problema de estudio o caso hipotético planteado.

\section{Entrevista retrospectiva}

- Consideran las ideas, destrezas y actitudes de sus estudiantes, al momento de plantear la resolución de un problema, de discutir un tema, de fijar una posición.

- Sienten temor de considerar las ideas que traen los estudiantes para realizar en el laboratorio porque cuando presentan alguna generalmente es por curiosidad, sin un sustento teórico y esto puede generar accidentes o situaciones inesperadas Consideración de ideas, destrezas y actitudes

Entrevista no estructurada

- Generalmente hay receptividad a los planteamientos realizados, ya que los ejercicios, problemas o situaciones hipotéticas planteadas por el docente son resueltas la mayoría de las veces con ayuda de lo que aportan los estudiantes

- En laboratorio se dan orientaciones a los estudiantes sobre el qué realizar y el cómo realizarlo. Ellos deben leer la práctica antes, por lo que se supone que deben saber como trabajar. El docente no está totalmente vigilante del trabajo del grupo, permite libertad aún cuando en ocasiones hace un monitoreo de cómo se está desarrollando la práctica 


\section{Uso de la bibliografía}

Los docentes involucrados en la investigación permiten que sus estudiantes revisen diversas fuentes bibliográficas con el objetivo de que la clase se haga más participativa y dinámica, haciendo de la misma un espacio en el que el estudiante participe también de la construcción de conocimientos (ver Figura 5). En este sentido, la documentación es importante al momento de abordar cualitativamente un problema de estudio, pues necesariamente debe tenerse un conocimiento base. Por ello, además de sugerir la revisión de los textos de la asignatura Química Orgánica, invitan a explorar el material adicional como son las guías de problemas y ejercicios, las diapositivas de presentaciones de power point, y material de Internet. Al reflexionar sobre el uso de las nuevas tecnologías en la enseñanza de la química, reconoce que Internet es un excelente medio para la búsqueda de información de temas concretos, pero que el estudiante puede creer que al disponer de la información tan fácilmente y "adornada" con excelentes dibujos y esquemas va a ser suficiente al momento de presentar trabajos y de documentarse.

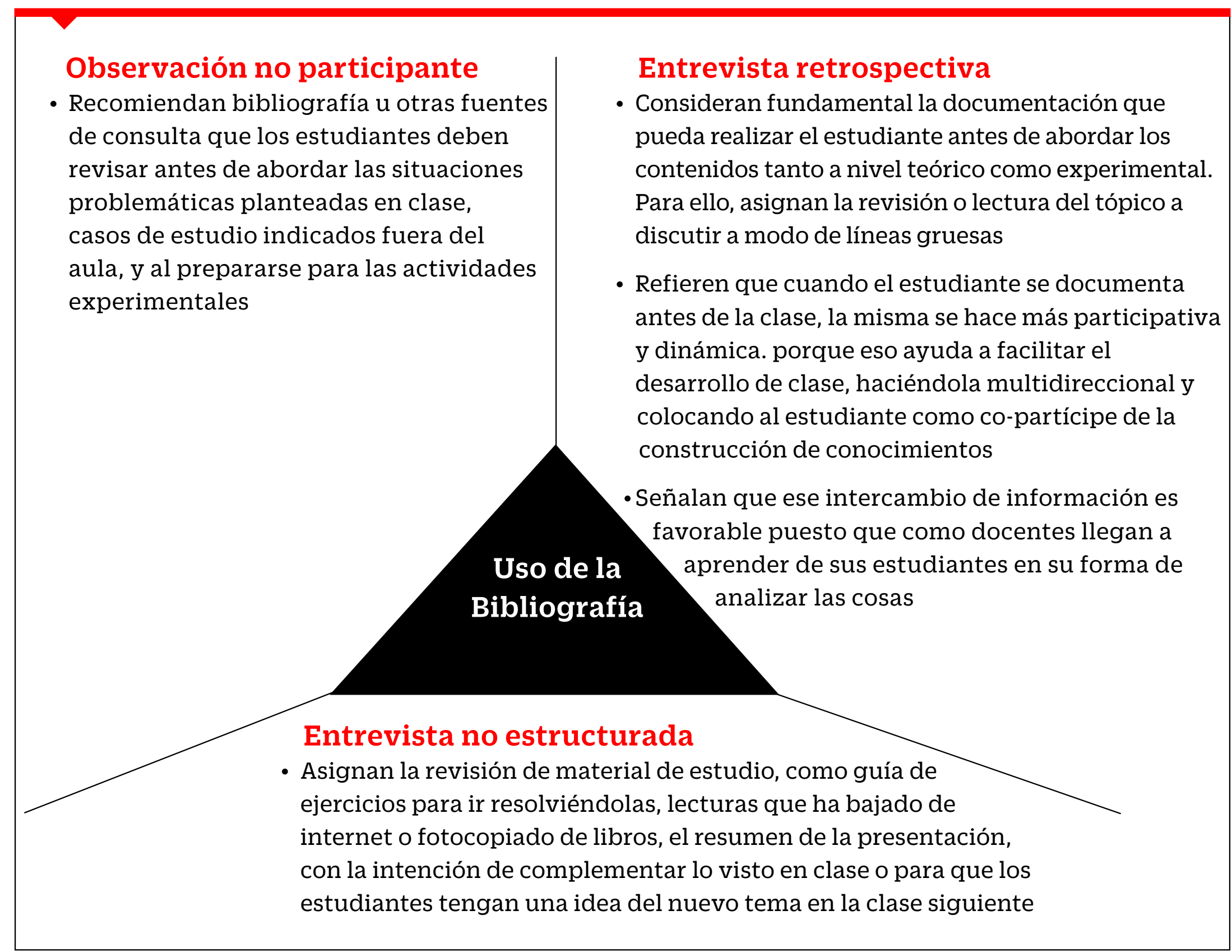

Figura 5. Categoría: Estudio Cualitativo de las situaciones problemáticas. Subcategoría: Uso de la bibliografía 
Sin embargo, cuando los docentes en cuestión solicitan la revisión bibliográfica del estudiante, estos no consideran que son copartícipes del proceso de construcción de conocimiento, pues asumen que la razón de esto es ayudarles a complementar lo visto en clase y que tengan noción de lo que se discutirá en una próxima sesión.

\section{Emisión de hipótesis}

Los docentes informantes clave inducen en sus estudiantes la formulación de hipótesis, pues se evidenció a través de la observación en los participantes que los mismos son capaces de emitir hipótesis frente a las situaciones de análisis y al momento de resolver problemas prácticos, para lo que deben tener noción de los principios científicos que gobiernan los fenómenos planteados. Por ello, con frecuencia deben estimular la emisión de hipótesis, dado que el estudiante raras veces suele hacerla por sí mismo, y es ahí donde converge la información de las entrevistas no estructuradas (ver Figura 6), pues en general el estudiante siente que la solución a los problemas es ofrecida por el docente, con poca participación de él. Así, lo anterior puede relacionarse con lo planteado por Poggioli, (1999) sobre el aprendizaje dirigido que se centra en la experiencia guiada por un experto -el docente- y el desarrollo de habilidades ocurre progresivamente gracias al modelamiento de la ejecución de la tarea, el uso de procedimientos propios de una ejecución experta y, por último, de la retroalimentación de la ejecución de los estudiantes con la finalidad de aproximarlos a tal nivel de destreza.

Sin embargo, en vista de que formular explicaciones justificadas, bien hipótesis que expresan una relación entre variables, o bien modelos que expresan su comprensión de la realidad, es una de las finalidades educativas de los procesos investigativos para la enseñanza de las ciencias (Martínez, Jiménez \& Lucio, 2015), se considera relevante que el docente enseñe a formularlas para evitar orientarlas: Una hipótesis es una suposición de algo posible o imposible para sacar de ello una consecuencia; y la hipótesis de trabajo es la que se establece provisionalmente como base de una investigación.

Cuando los estudiantes generan hipótesis es porque han desarrollado algunos procesos investigativos, tales como sobre la base de un problema -ofrecido generalmente por el docente en torno a la explicación de algún fenómeno o resolución de problemas/ejercicios en el transcurso de la clase- observan su naturaleza y proceden a analizar y seleccionar lo relevante, reuniendo todos los datos que ofrece para su resolución. Otro proceso es el que se desarrolla una vez recogidos los datos, pues el estudiante está en capacidad de elaborar una explicación provisional que describa de la forma más simple posible las razones que generan el problema en cuestión, o la solución del mismo, tal como un enunciado breve o una formulación matemática, respectivamente. 
Observación no participante

- Los estudiantes tienden a emitir hipótesis. Las mismas suelen ser espontáneas, pero en algunos casos son orientadas con la ayuda del profesor, quien va en su discurso ofreciendo pistas para que los estudiantes vislumbren el emitir algún juicio.

\section{Entrevista retrospectiva}

- Indican que sus estudiantes generan hipótesis por si solos, pero es gracias a que ya traen una noción previa por lo que han leído, por la documentación. Estas hipótesis están relacionadas muchas veces con las dudas que se presentan en las clases sobre la resolución de problemas.

- Reconoce que por lo general necesitan de la orientación ya que hay que decir hacia dónde debe estar dirigida la resolución, el análisis que deben hacer

\section{Entrevista no estructurada}

- Pocas veces el estudiante pasa a resolver un problema en la pizarra en el que tenga que generar hipótesis. En general las situaciones problemáticas de cualquier índole son resueltas por el docente, y de ahí los estudiantes toman las ideas.

Figura 6. Categoría: Estudio Cualitativo de las situaciones problemáticas. Subcategoría: Emisión de hipótesis

\section{Elaboración de estrategias de resolución}

Los docentes permiten -a su criterio-elaborar la vía de resolución de las situaciones planteadas; sin embargo, generalmente lo que se propone en clases son ejercicios donde los datos están totalmente explícitos y lo que "falta" es generar la solución a manera de rompecabezas. No se tratan entonces de problemas reales, pues los medios o caminos de resolución son evidentes. Esto es propio de los enunciados clásicos de materias de ciencias, como es el caso de química, que al exponer todos los datos necesarios para la resolución e indicar qué modelos matemáticos se deben utilizar, no son más que ejercicios de reconocimiento de un algoritmo ya utilizado por el profesor para resolverlo, o búsqueda de fórmulas en qué sustituir todos los datos y despejar la incógnita (ver Figura 7).

Por otra parte, en el ámbito experimental, los estudiantes siguen las pautas dictadas por el manual de laboratorio, por tanto no hay situaciones verdaderamente problemáticas que demanden de ellos procesos heurísticos o de pensamiento divergente para resolverlos, como los planteados por Poggioli (1999). 
Observación no participante

- Permiten a sus estudiantes tomar decisiones sobre lo que van a investigar, mientras no distorsionen aspectos importantes de la asignatura como los objetivos de la clase y del contenido, y se apeguen al contexto en estudio

\section{Entrevista retrospectiva}

- Permiten al estudiante elaborar la vía de resolución de problemas en la clase teórica, ya que cada estudiante puede tener una visión distinta con la cual abordar los problemas, y eso enriquece la clase, evita la sistematización y unificación de criterios

- Indican que los estudiantes no elaboran la vía de resolución a los experimentos planteados, ya que siempre se les suministra a través de lo escrito en los manuales prácticos de laboratorio. En tal caso, lo que se les pide es esquematizar el procedimiento, y ahí se aprecia si han comprendido lo que van Elaboración de estrategias a realizar durante la práctica

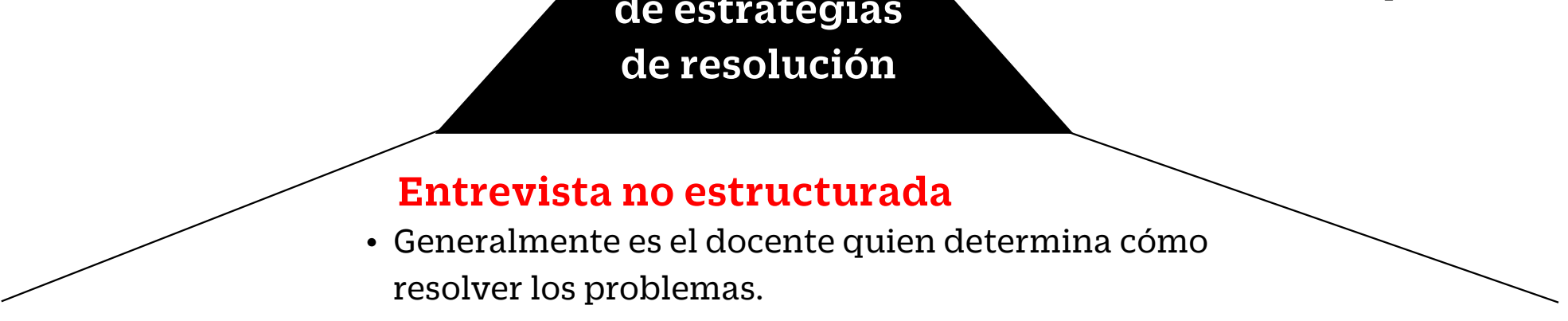

- Se siguen las instrucciones de la práctica, y los estudiantes no recuerdan que se les haya pedido idear un procedimiento experimental, o que se planteen el estudio de algo que no esté en la guía de laboratorio, donde sean ellos los que decidan que materiales y reactivos se van a utilizar.

- Lo que se va es a comprobar lo que dice la teoría .

Figura 7. Categoría: Estudio Cualitativo de las situaciones problemáticas. Subcategoría: Elaboración de estrategias de resolución

\section{DISCUSIÓN}

Los docentes informantes clave poseen una concepción del proceso de investigación muy similar a la que establece la literatura. Sin embargo, su desarrollo en la enseñanza de la Química Orgánica es débil dentro del contexto de teórico por cuanto el estudiante solo lee y revisa el material dispuesto por el docente; y en el contexto de laboratorio, la investigación se limita a revisar los aspectos teóricos correspondientes a la práctica por desarrollar, para ejecutar una prueba de prelaboratorio. Lo anterior no se condice con una forma sistemática de aproximación al conocimiento a través de auténticas actividades que favorezcan la 
comprensión y evolución de las ideas científicas. En alusión particular a los trabajos prácticos, no se fomentan actividades de investigación desde una propuesta problematizadora, caracterizándose por ser de verificación de la teoría, con poca o ninguna relación con los contenidos que se desarrollan en las clases teóricas. Esto es relevante en tiempos donde los enfoques de indagación científica, las estrategias como el aprendizaje basado en problemas, o los modelos didácticos de enseñanza por investigación están presentes en la literatura de didáctica de las ciencias, y son demandados desde la educación secundaria al profesorado en formación, donde «la deficiente formación didáctica que tienen, en general, los profesores de ciencias de las facultades (y que, dicho sea de paso, no suelen reconocer) la han transmitido a sus alumnos (profesores de secundaria) y estos, a su vez a sus alumnos» (Cartaña \& Comás, como se citaron en Campanario, 2002).

Por ello, proponer actividades a través de las cuales se promueva la investigación, tales como problemas abiertos y casos experimentales que demanden del estudiante la búsqueda, síntesis, análisis, estrategias de resolución, entre otras habilidades cognitivas y cognitivo-lingüísticas.

Las ideas de los estudiantes son consideradas una vez que el docente plantea lo que considera un "problema" -el cual generalmente ofrece todos los datos para su resolución, haciéndolo altamente predecible, algorítmico- en el esbozo de su posible solución, pero no en la construcción de las situaciones problemáticas por discutir durante la clase -ni en las que se puedan ejecutar en el laboratorio por temor a accidentes inesperados.

En el caso revisado se fomenta la discusión de la literatura en el aula, tomando como base lo que sus estudiantes revisan de la bibliografía para hacer la clase más dinámica y participativa. Sin embargo, hay el riesgo de que 1) el estudiante revise solo un tipo de fuente como lo es la provista por la plataforma tecnológica web, que presenta la información en ocasiones de manera poco seria y tergiversada; 2) al no revisar otras fuentes como textos o revistas especializadas, el estudiante pierde la oportunidad de comparar la información recopilada y llegar a conclusiones más elaboradas y potentes; y por último 3) el estudiante realice un pobre procesamiento de la información debido a una lectura incompleta del material indicado por el docente antes de discutir el contenido, o que sencillamente no lo revise. Por esto, sería idóneo que el docente diseñe actividades en las que se oriente el uso de la bibliografía a través de cuestiones de interés que ayuden al desarrollo investigativo.

Adicionalmente, se permite la elaboración de hipótesis en la resolución de problemas de lápiz y papel durante los abordajes teóricos por parte de los estudiantes, que al ser dirigida por el docente, subestima la habilidad del estudiante. Esto ocurre únicamente en las clases teóricas, pues como se observó en el laboratorio generalmente no se tiene la posibilidad de conjeturar debido a su carácter pre-establecido: los resultados se conocen.

Los docentes permiten en ocasiones elaborar la vía de resolución de los ejercicios que plantean en clases, mientras que, en el ámbito experimental, diseñar una vía de resolución al 
problema de estudio parece paradójico, pues en líneas generales, el laboratorio no representa una situación problemática sobre la cual trabajar.

Durante las clases, los docentes permiten a sus estudiantes confrontar los resultados obtenidos de la resolución de ejercicios para que estos verifiquen si están correctos o no. En el Laboratorio ocurre una situación contraria, debido a la falta de tiempo que, como señalaron los docentes, apremia inclusive el logro completo de la parte experimental, por lo que se minimizan las posibilidades de transferencias que los estudiantes puedan hacer con el nuevo conocimiento. Confrontar permanentemente los resultados de los trabajos experimentales, de manera que se comparta el conocimiento y se oriente el proceso de retroinformaciónregulación de lo aprendido y su transferencia por la reiteración en variedad de situaciones, es un ideal precisado.

De esta forma, la implementación de la investigación para la enseñanza de la química en la formación inicial docente del caso estudiado dista de las formas y finalidades que la literatura en Didáctica de las Ciencias Experimentales aporta en la actualidad. Se reconoce un matiz tradicionalista en la enseñanza de estos contenidos en el caso, que suele ser un modelo a replicar por parte del profesorado en la enseñanza media, una vez finalizada la formación. Por ello, se recomienda continuar revisando lo que sucede en la docencia universitaria de la disciplina que participa de los programas de formación inicial, ya que Galiano y Sevillano (2015) advierten que el estudiante de profesorado en química debe ser capaz de aprender a preguntar, cuestionar, generar problemas y respuestas provisorias, revisar los conocimientos químicos a la luz de los marcos teóricos vigentes en un momento de la historia. Estos procesos reflexivos son los caminos que deberán recorrer en su formación.

\section{REFERENCIAS}

Adúriz-Bravo, A. (2011). Desde la enseñanza de los "productos de la ciencia" hacia la enseñanza de los "procesos de la ciencia" en la Universidad". Colección de Cuadernillos de Actualización para Pensar la Enseñanza Universitaria, 6(3), 5-12.

Álvarez-Gayou, J. (2009). Cómo hacer investigación cualitativa. Fundamentos y Metodología. México: Paidós Educador. Belmonte, M. (2011). Enseñar a investigar. Libro del profesorado. Bilbao: Mensajero.

Burbules, N. \& Linn, M. (1991). Science education and philosophy of science: congruence or contradiction? International Journal of Science Education, 13(3), 227-241. http://dx.doi.org/10.1080/0950069910130302

Caamaño, A. (2012). ¿Cómo introducir la indagación en el aula? Alambique. Didáctica de Las Ciencias Experimentales, $70,83-91$ 
Campanario, J. M. (2002). Asalto al castillo: ¿A qué esperamos para abordar en serio la formación didáctica de los profesores universitarios de ciencias? Enseñanza de las Ciencias: Revista de Rnvestigación y Experiencias Didácticas, 20(2), 315-326. Recuperado de https://goo.gl/Hm71ed

Canales, M. (2006). Metodologías de la investigación social. Introducción a los oficios. Santiago: LOM

Couso, D. (2002) La comunidad de Aprendizaje Profesional: Una propuesta socioconstructivista de desarrollo profesional del profesorado de ciencias naturales. En A. Perafán \& A. Adúriz-Bravo (Eds.), Pensamiento y conocimiento de los profesores: Debate y perspectivas internacionales (2a ed.) (pp. 81-100). Bogotá: Universidad Pedagógica Nacional.

Cuéllar, L. (2010). La Historia de la Química en la Reflexión sobre la Práctica Profesional Docente (Tesis doctoral). Recuperado de https://goo.gl/v9dH7a

Dezin, N \& Lincoln, D. (2012). Paradigmas y perspectivas en disputa. Manual de investigación cualitativa II. Brcelona: Gedisa

Ferrés, C., Marbà, A. \& Sanmartí, N. (2015). Trabajos de indagación de los alumnos: Instrumentos de evaluación e identificación de dificultades. Revista Eureka sobre Enseñanza y Divulgación de las Ciencias, 12(1), 22-37. Recuperado de https://goo.gl/32rgDw

Flick, U. (2004). Introducción a la investigación cualitativa. Madrid: Morata.

Galiano, J. \& Sevillano, M. (2015). Estrategias de enseñanza de la Química en la formación inicial del Profesorado Universitario. Educatio Siglo XXI, 33(1), 215-234. doi: https://doi.org/10.6018/j/222571

García, A. (2009). Aportes de la Historia de la Ciencia al desarrollo profesional de los profesores de Química (Tesis doctoral). Universitat Autónoma de Barcelona, Barcelona.

García, M. (2000). Desarrollo de habilidades, destrezas y hábitos, objetivo del laboratorio de química en la educación media diversificada. Boletín CENAMEC Multidisciplinario, 9, 27-63.

Garritz, A. \& Velazco, R. (2004). El Conocimiento Pedagógico del Contenido. Educación Química, 15(2), 2-6. Recuperado de https://goo.gl/KvzK6z

Gil, D. (1993). Contribución de la Historia y de la Filosofía de las Ciencias al desarrollo de un modelo de enseñanza/ aprendizaje como Investigación. Enseñanza de las Ciencias, 11(2), 197-212. Recuperado de https://goo.gl/ Ch7d2u

Jiménez-Tenorio, N. \& Oliva, J. (2016). Aproximación al estudio de las estrategias didácticas en ciencias experimentales en formación inicial del profesorado de Educación Secundaria: descripción de una experiencia. Revista Eureka sobre Enseñanza y Divulgación de las Ciencias, 13(1), 121-136. Recuperado de https://goo.gl/pW1mbN

Martínez, M., Jiménez, M. \& Lucio, R. (2015). Efecto de un programa formativo para enseñar ciencias por indagación basada en modelos, en las concepciones didácticas de los futuros maestros. Revista Eureka sobre Enseñanza y Divulgación de las Ciencias, 12 (1), 149-166. Recuperado de https://goo.gl/Gjuu44

Mellado, V. \& González, T. (2000). La Formación Inicial del profesorado de Ciencias. En F. Perales \& P. Cañal, (Eds.), Didáctica de las Ciencias Experimentales (pp. 535-556). Valencia: Marfil.

Neiman, G. \& Quaranta, G. (2006). Los estudios de caso en la investigación sociológica. En I. de Gialdino Vasilachis (Ed.), Estrategias de investigación cualitativa (pp. 213-238. Buenos Aires: Gedisa.

Poggioli, L. (1999). Enseñando a Aprender. Venezuela: Ediciones Fundación Polar.

Pozo, J. \& Gómez, M. (2013). Aprender y Enseñar Ciencia. Madrid: Morata.

Simons, H (2011). El estudio de caso: teoría y práctica. Madrid: Morata.

Ungerfeld, R. (2004). La investigación como soporte de actividades de enseñanza universitaria. Contexto educativo: revista digital de investigación y nuevas tecnologías, 31, 5. 
Vázquez, Á. \& Manassero, M. (2012). La selección de contenidos para enseñar naturaleza de la ciencia y tecnología (parte 1): Una revisión de las aportaciones de la investigación didáctica. Revista Eureka sobre Enseñanza y Divulgación de las ciencias, 9(1), 2-31. Recuperado de https://goo.gl/sGkk42

Vázquez, B., Jiménez, R. \& Mellado, V. (2007). El desarrollo profesional del profesorado de ciencias como integración de la reflexión y la práctica. La Hipótesis de la Complejidad. Revista Eureka sobre Enseñanza y Divulgación de las Ciencias, 4(3), 372-393. Recuperado de https://goo.gl/UP8Kux

RIDU / Revista Digital de Investigación en Docencia Universitaria / ISNN 2223-2516

( ) Los autores. Este artículo es publicado por la Revista Digital de Investigación en Docencia Universitaria del Área de Institutional Research and Effectiveness de la Dirección de Aseguramiento de la Calidad, Universidad Peruana de Ciencias Aplicadas. Este es un artículo de acceso abierto, distribuido bajo los términos de la LicenciaCreativeCommons Atribución-CompartirIgual 4.0 Internacional. ( http://creativecommons.org/licenses/by-sa/4.o/), que permite el uso no comercial, distribución y reproducción en cualquier medio, siempre que la obra original sea debidamente citada. 